\title{
Role of elastic scattering in electron dynamics at ordered alkali overlayers on $\mathrm{Cu}(111)$
}

\author{
C. Corriol, ${ }^{1}$ V. M. Silkin, ${ }^{1}$ D. Sánchez-Portal,,${ }^{1,2}$ A. Arnau, ${ }^{1,2,3}$ E. V. Chulkov, ${ }^{1,2,3}$ P. M. Echenique ${ }^{1,2,3}$ \\ ${ }^{1}$ Donostia International Physics Center (DIPC), \\ ${ }^{2}$ Centro Mixto C.S.I.C.-UPV/EHU, and ${ }^{3}$ Departamento de Física de Materiales, \\ Facultad de Química, Apartado 1072, San Sebastian 20080, Spain \\ T. von Hofe, J. Kliewer * J. Kröger, and R. Berndt \\ Institut für Experimentelle und Angewandte Physik, \\ Christian-Albrechts-Universität zu Kiel, D-24098 Kiel, Germany
}

\begin{abstract}
Scanning tunneling spectroscopy of $\mathrm{p}(2 \times 2) \mathrm{Cs}$ and $\mathrm{Na}$ ordered overlayers on $\mathrm{Cu}(111)$ reveals similar line widths of quasi two-dimensional quantum well states despite largely different binding energies. Detailed calculations show that $50 \%$ of the line widths are due to electron-phonon scattering while inelastic electron-electron scattering is negligible. A frequently ignored mechanism for ordered structures, i.e., enhanced elastic scattering due to Brillouin zone back folding, contributes the remaining width.

PACS numbers: 73.20.At, 68.37.Ef, 73.21.Fg, 71.20.Gj
\end{abstract}

In many electron systems ubiquitous interactions between quasiparticles such as electron-electron, electronphonon or electron-magnon scattering are present and cause inelastic scattering. A fingerprint of this dynamics, which occurs on a femtosecond timescale, is the width of spectroscopic lines. Often, elastic scattering is significant too, except for well defined surface states at special points in the surface Brillouin zone (SBZ). Thus, surface resonances exhibit a finite width owing to a degree of mixing with the bulk continuum. Defect scattering can also increase the line width of electron states at artificial atomic arrays or at electron confining island [1, 2, 3, 4]. Adsorbates on a surface lead to additional scattering channels that may change the dynamics of electrons at surfaces and at the same time can be an important source of elastic scattering for electrons 5]. An intriguing situation occurs when an extended ordered superstructure changes the periodicity of a surface, as in the case of submonolayer coverages of alkali atoms on (111) noble metal surfaces. The SBZs of the substrate and of the overlayer have different sizes. The resulting back folding of the bands can lead to large effects in the dynamics of electrons at the interface, like the appearance of an elastic width of quantum well states (QWS) when energy gaps are closed in certain regions of the SBZ.

Inverse photoemission and two-photon photoemission are widely used techniques to probe the dynamics of hot electrons at surfaces [6, 7]. Recently, the different mechanisms that contribute to the elastic and inelastic scattering of hot electrons at $\mathrm{Cu}(001)$ surfaces with a low coverage of $\mathrm{Cu}$ adatoms have been identified [8] using these techniques. The conclusions are deduced from information taken over the whole surface area without probing the local electronic structure. Scanning tunneling spectroscopy (STS) resolves the local electronic structure of surface areas free from defects. It also can provide detailed information about both occupied and unoccupied states. The lifetime broadening of surface states can be extracted from STS measurements by doing a line shape analysis 9, 10, 11, 12 or from electron standing wave patterns [13, 14, 15]. The progress of these experimental approaches clearly requires refined theoretical methods if one aims to explain experimental data at a quantitative level.

Here, in a combined experimental and theoretical study, we analyze the processes that contribute to the width of QWSs at $\mathrm{Cs}$ and $\mathrm{Na}$ overlayers on $\mathrm{Cu}(111)$. We achieve quantitative agreement between low temperature STS experiments and simulations of tunneling spectra using first principles calculations of the electronic structure of the surface and of the line width. The width of the QWS is shown to have significant contributions from the elastic width that appears as a result of the coupling to substrate states and from the inelastic electron-phonon scattering whereas inelastic electron-electron interaction is of minor importance, contrary to the situation observed in studies of the hole dynamics at the (111) surface of noble metals 10 .

Clean $\mathrm{Cu}(111)$ surfaces were prepared by standard $\mathrm{Ar}^{+}$ bombardment and annealing cycles. Cs and $\mathrm{Na}$ were deposited from commercial dispensers [16] onto the sample at room temperature at rates of $\approx 0.5 \mathrm{ML} \mathrm{min}^{-1}$ as monitored with a quartz microbalance. We define one monolayer (ML) as one alkali atom per one $\mathrm{Cu}$ atom of the unreconstructed $\mathrm{Cu}(111)$ substrate. In the Cs case, a clear $\mathrm{p}(2 \times 2)$ low-energy electron diffraction $($ LEED) pattern at room temperature [17, 18] was used as additional indication of a $0.25 \mathrm{ML}$ coverage. After preparation, samples were transfered to a scanning tunneling microscope (STM) and cooled to temperatures of $4.6-9$ K. Spectroscopy of the differential conductance $(\mathrm{d} I / \mathrm{d} V)$, was performed on homogeneous areas using a lock-in amplifier and adding a sinusoidal modulation $\left(6-14 \mathrm{mV}_{\mathrm{pp}}\right.$, $10 \mathrm{kHz}$ ) to the sample voltage. 
Figure 1 displays $\mathrm{d} I / \mathrm{d} V$-spectra (dots) of $\mathrm{p}(2 \times 2)$ Cs and Na layers. Sharp rises of the conductance at $E_{0} \cong 40 \mathrm{meV}$ and $E_{0} \cong 410 \mathrm{meV}$ correspond to the minima of the quantum well state bands of $\mathrm{Cs}$ and $\mathrm{Na}$ overlayers, respectively. The width of the rise, which is related to the inverse lifetimes of the these states [9], is similar in both cases. This observation is surprising in view of the large difference in binding energy which, in a simple picture, should cause widely different electronelectron $(e-e)$ scattering. However, this appears not to be the case. Our calculations, whose results agree well with the experimental data (solid lines in Fig. 1), show that inelastic $e-e$ scattering is unimportant and that electron-phonon $(e-p h)$ scattering explains only $50 \%$ of the line widths. Enhanced elastic scattering due to Brillouin zone back folding, turns out to contribute the missing $50 \%$ of the line width.

The surface electronic structure of both systems has been calculated using the density functional code VASP [19]. Projected Augmented Waves [20] are used to describe the ionic cores and a plane wave basis set for the valence electrons. The exchange and correlation potential is treated in the generalized gradient approximation 21]. We have checked the convergency of the calculations with respect to the thickness of the slab up to 10 layers of $\mathrm{Cu}$. The $k$-point sampling of the irreducible wedge of the surface Brillouin zone of the $(2 \times 2)$ superstructure is done using 14 special points for the self-consistent calculation and later on we use up to 154 points to achieve high enough resolution of the bands. These slab calculations provide the structure for the recursive calculation of the elastic width in the semi-infinite medium. The tunneling conductance is calculated with a model that assumes a smooth density of states of the tip around the Fermi level. Then, at small positive sample bias, the energy dependence of the conductance is determined by the sample density of states 22]. The details of the model are described elsewhere 23]. In brief, we introduce an energy width $\left(\Gamma_{m} / 2\right)$ of the states when calculating the imaginary part of the surface Green function, i.e., the surface local density of states (LDOS). This width corresponds to a Lorentzian weight for each state such that the surface LDOS is given by:

$$
\rho(\varepsilon, \vec{r})=\frac{1}{\pi} \sum_{m}\left|\Psi_{m}(\vec{r})\right|^{2} \frac{\Gamma_{m} / 2}{\left(\varepsilon-\varepsilon_{m}\right)^{2}+\left(\Gamma_{m} / 2\right)^{2}}
$$

The eigenvalues $\left(\varepsilon_{m}\right)$ and eigenfunctions $\left(\Psi_{m}\right)$ are taken from VASP, while the energy width $\left(\Gamma_{m} / 2\right)$ is determined as described below. The sum over $m$ includes both a summation over the band index and an integration over parallel momentum. The LDOS is calculated at $3 \AA$ from the alkali overlayer, where the electronic corrugation is negligible, and it is dominated by the QWS band in the relevant energy range. A $k$-point interpo-
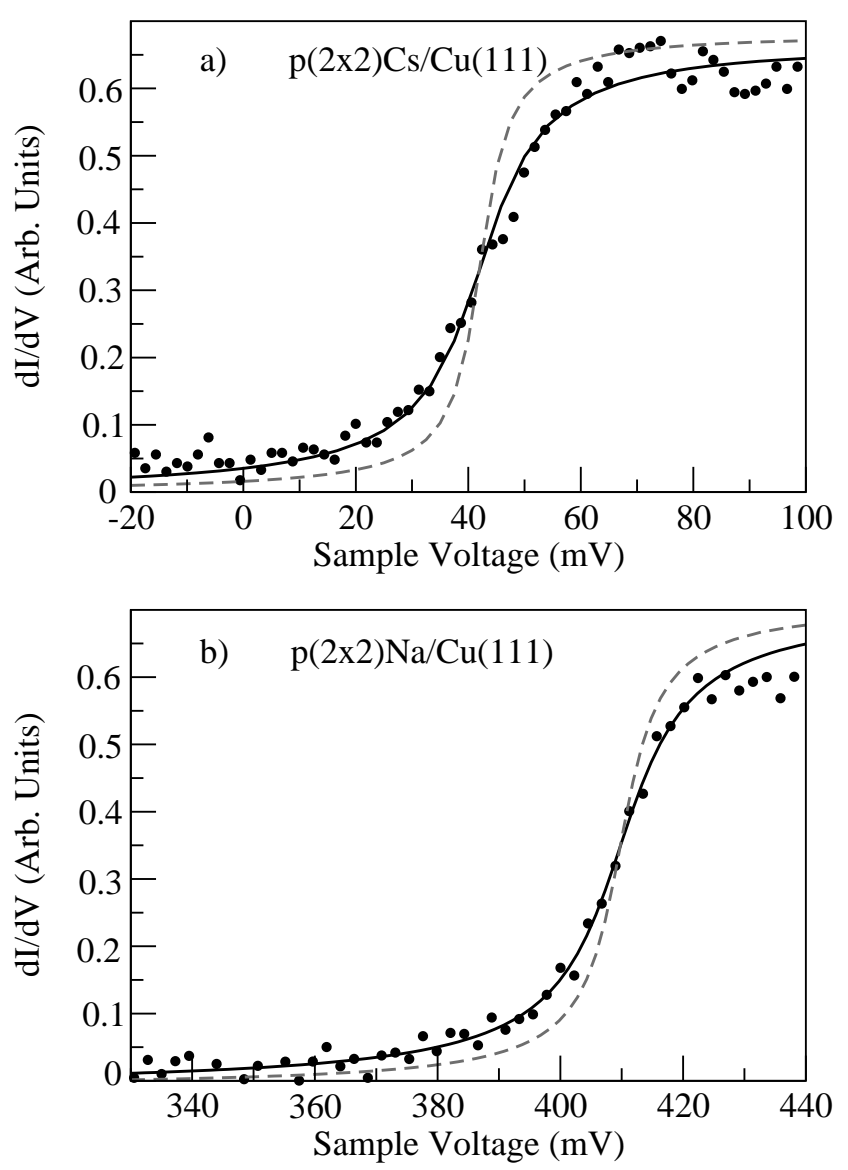

FIG. 1: Comparison between measured $\mathrm{d} I / \mathrm{d} V$ vs. $V$ curves (dots) for $\mathrm{p}(2 \times 2)$ structures of (a) Cs and (b) $\mathrm{Na}$ on $\mathrm{Cu}(111)$ along with calculated results (lines). The solid lines include both the elastic and inelastic contributions to the energy width, while the dashed lines include only the inelastic one.

lation of the QWS is used to achieve the required meV resolution of the band 23].

For comparing the measured $\mathrm{d} I / \mathrm{d} V$ data for the $\mathrm{p}(2 \times$ 2) Cs structure on $\mathrm{Cu}(111)$ and the simulated spectrum we return to Fig. 17. No fitting parameter has been used apart from a scaling factor for the calculated conductance. The agreement between the data points and the solid curve clearly shows the importance of the elastic width in determining the shape of the spectrum at the onset around $40 \mathrm{meV}$. Fig. 1b displays a similar comparison for a $\mathrm{p}(2 \times 2) \mathrm{Na}$ film on $\mathrm{Cu}(111)$. In this case, the calculated curves have been shifted by $+18 \mathrm{meV}$ to match the experimental binding energy. This small shift is consistent with the expected accuracy of density functional calculations 24]. The shape of the curve at the onset also agrees well with the measured data in this case.

The adsorption of alkali adlayers in $\mathrm{p}(2 \times 2)$ superstructures leads to a modification of the projected bulk band structure of the substrate as shown in Fig. 2 along the $\bar{\Gamma}-\overline{\mathrm{M}}$ direction. The inset displays the SBZs for a 
$\mathrm{p}(2 \times 2)$ overlayer and for a $(1 \times 1)$ substrate. In the overlayer case the energy gap near $E_{F}$ of clean $\mathrm{Cu}(111)$ surface is closed due to the folding of bands originally situated along the $\overline{\mathrm{M}}-\overline{\mathrm{M}}_{\mathrm{Cu}}$ and $\overline{\mathrm{M}}_{\mathrm{Cu}}-\overline{\mathrm{K}}^{\prime}$ symmetry directions. The corresponding extra corrugation of the $\mathrm{Cu}$ (111) surface due to the coupling between the $\mathrm{p}(2 \times 2)$ adsorbate layer and underlying $\mathrm{Cu}$ bulk states causes a degree of mixing between the alkali induced QWS at $\bar{\Gamma}$ and substrate $\mathrm{Cu}$ states at the $\overline{\mathrm{M}}_{\mathrm{Cu}}$ point of the $(1 \times 1)$ Brillouin zone. This serves as a source of elastic scattering, which requires a quantitative analysis.

To calculate the elastic width of QWSs we compute the Green function of the combined system (adlayer plus semi-infinite substrate) with high precision in the surface region. Here, we use the SIESTA code 25] which utilizes a basis set of numerical atomic orbitals 26 . The results for the electronic structure are almost identical to those obtained with VASP. With this local basis set the elements of the Hamiltonian and overlap matrices between atoms that are far apart (beyond $\approx 15 \AA$ in our case) are strictly zero. The infinite system can now be divided in groups of layers ("slices") that only interact with the nearest neighbor groups. The Hamiltonian matrix elements (and overlaps) for orbitals in the surface slice, and its interaction with orbitals in underlying layers, are obtained from the slab calculation. The interactions in the inner slices are taken from a calculation of bulk $\mathrm{Cu}$. A common energy reference is set by aligning the Fermi levels of both calculations. We now use the recursive relation

$$
G^{i j}\left(\omega, \mathbf{k}_{\|}\right)\left[H_{j k}\left(\mathbf{k}_{\|}\right)-\omega S_{j k}\left(\mathbf{k}_{\|}\right)\right]=\delta_{k}^{i}
$$

to obtain the Green function in the surface region. We use a method similar to that described in Ref. 27] to iteratively solve this equation. The matrix elements are defined such that $G\left(\mathbf{r}, \mathbf{r}^{\prime} ; \omega, \mathbf{k}_{\|}\right)=G^{i j}\left(\omega, \mathbf{k}_{\|}\right) \phi_{i}(\mathbf{r}) \phi_{j}\left(\mathbf{r}^{\prime}\right)$, where the $\phi_{i}$ are the atomic orbitals and the sum over repeated indices is assumed. Our strategy is then to project the Green function onto a wave packet $\Psi_{R}$ localized in the surface region. The function $\operatorname{Im}\left\{<\Psi_{R}|\hat{G}(\omega)| \Psi_{R}>\right\}$ exhibits well defined peaks at energies $\epsilon_{R}$ corresponding to the QWS of the overlayer. The shape and position of these peaks are independent of the election of $\Psi_{R}$, as far as $\Psi_{R}$ has predominant contributions from the orbitals in the adsorbates. The peaks have a Lorentzian shape with a width and position that can be easily extracted using a least square fit 28]. Carrying out this procedure for the two systems studied here we find values of the elastic width at $\bar{\Gamma}$ equal to $9.4 \mathrm{meV}$ and $7.4 \mathrm{meV}$ for $\mathrm{Cs}$ and Na systems, respectively.

For both systems of interest we estimate the inelastic electron-electron $(e-e)$ contribution $\left(\Gamma_{e-e}\right)$ to the QWS linewidth by using a model in which the one-electron potential is constant in a plane parallel to the surface and varies only in the direction $z$ perpendicular to the surface. We use here the potential proposed in Ref. 29]

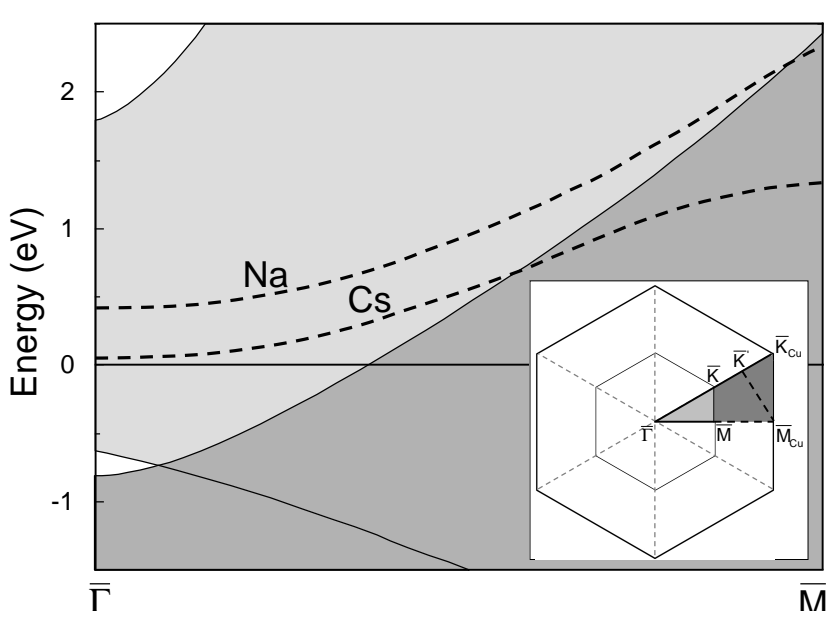

FIG. 2: Dispersion of $\mathrm{p}(2 \times 2) \mathrm{Na}$ and Cs quantum well states in the $\bar{\Gamma}-\overline{\mathrm{M}}$ direction. Dark grey indicates bulk $\mathrm{Cu}$ states of the $(1 \times 1)$ SBZ, while light grey shows the closing of the gap around $\bar{\Gamma}$ after back folding from the $\overline{\mathrm{M}}-\overline{\mathrm{M}}_{\mathrm{Cu}}$ and $\overline{\mathrm{M}}_{\mathrm{Cu}}-\overline{\mathrm{K}}^{\prime}$ symmetry directions defined in the inset.

for thin films adsorbed on a $\mathrm{Cu}(111)$ substrate with parameters fitted to reproduce the binding energy of the QWS in the Cs $(42 \mathrm{meV})$ and $\mathrm{Na}(408 \mathrm{meV})$ adlayer on $\mathrm{Cu}(111) . \Gamma_{e-e}$ is calculated within the GW approximation [7] by employing eigenfunctions and eigenenergies obtained with this model potential. The computed $\Gamma_{e-e}$ values of less than $0.1 \mathrm{meV}$ and $0.4 \mathrm{meV}$ for a QWS in the $\mathrm{Cs}$ and $\mathrm{Na}$ systems, respectively, demonstrate that inelastic $e-e$ scattering provides only a small contribution to the QWS linewidth. This latter fact reflects the difference between electrons and holes: for electrons at the bottom of the QWS the intraband channel is completely absent, while it is most important for holes [10].

Another inelastic contribution, the electron-phonon $(e-p h)$ one, is found to be important for the studied systems. While there is no detailed information on phonon dispersion of these systems, one can refer to helium atom scattering measurements for a monolayer of $\mathrm{Cs}$ on $\mathrm{Cu}(100)$ [30]. Assuming that this phonon structure is also valid for $\mathrm{Cs}$ on $\mathrm{Cu}(111)$ we evaluate $\Gamma_{e-p h}$ by using the Einstein model 31] to treat a flat phonon mode with an energy of $7.5 \mathrm{meV}$ around the $\bar{\Gamma}$ point and the Debye model 31] for the two acoustic modes (Rayleigh and longitudinal ones) with maximum energy of $6 \mathrm{meV}$. The $e-p h$ coupling parameter $\lambda=0.18$ is taken from Ref. 31] as the maximum bulk value for $\lambda$. With this we estimate $\Gamma_{e-p h}=7.5 \mathrm{meV}$. For $\mathrm{Na}$ on $\mathrm{Cu}$ we also assume that the measured maximum phonon frequency for $0.44 \mathrm{ML}$ of $\mathrm{Na}$ on $\mathrm{Cu}(100)$ can be applied to $\mathrm{p}(2 \times 2)$ $\mathrm{Na} / \mathrm{Cu}(111)$. Following Ref. [29] we use $\lambda=0.24$ and Debye energy $\hbar \omega_{D}=18 \mathrm{meV}$ yielding $\Gamma_{e-p h}=9.0 \mathrm{meV}$.

An overview of the different contributions to the line width of the QWS at the $\bar{\Gamma}$ point is presented in Table 


\begin{tabular}{c||c|c|c|c} 
& $\Gamma_{\text {elastic }}$ & $\Gamma_{e-e}$ & $\Gamma_{e-p h}$ & $\Gamma_{\text {total }}$ \\
\hline \hline $\mathrm{Cs}$ & 9.4 & $<0.1$ & 7.5 & 17 \\
\hline $\mathrm{Na}$ & 7.4 & 0.4 & 9 & 16.8
\end{tabular}

TABLE I: Line width contributions (in meV) of various scattering channels. $\Gamma_{\text {elastic }}$ elastic, $\Gamma_{e-e}$ electron-electron, and $\Gamma_{e-p h}$ electron-phonon scattering. $\Gamma_{\text {total }}$ is the resulting total width.

【1 The elastic contribution to the energy width accounts for approximately $50 \%$ of the total width. The inelastic $e-p h$ contribution is comparable. However, the inelastic $e-e$ contribution is negligible.

In summary, our combined experimental and theoretical study shows that the dynamics of electrons at ordered overlayers is determined by the elastic width that appears due to the closing of gaps after back folding the bands and by inelastic e-ph scattering. However, inelastic e-e scattering does not play a role. The relative importance of the elastic contribution for other ordered superstructures will depend on details like the density of states at given points of the substrate SBZ. Any change of periodicity on going from the surface to the overlayer structure will lead to a modification of the scattering channels that determine the dynamics of electrons at the interface.

The Kiel authors thank the Deutsche Forschungsgemeinschaft. The San Sebastian authors thank Eusko Jaurlaritza, Euskal Herriko Unibertsitatea, the spanish M.E.C. and the EC grant NANOQUANTA for financial support.

* Present address: Infineon Technologies AG, D-81609 München, Germany

[1] E. J. Heller, M. F. Crommie, C. P. Lutz, and D. M. Eigler, Nature 369, 464 (1994).

[2] M. F. Crommie, C. P. Lutz, and D. M. Eigler, Science 262, 218 (1993).

[3] S. Crampin, M. H. Boon, and J. E. Inglesfield, Phys. Rev. Lett. 73, 1015 (1994).

[4] H. Jensen, J. Kröger, R. Berndt, and S. Crampin, accepted for publication in Phys. Rev. B

[5] A. G. Borisov, J. P. Gauyacq, A. K. Kazansky, E. V. Chulkov, V. M. Silkin, and P. M. Echenique, Phys. Rev. Lett. 86, 488 (2001).

[6] H. Petek and S. Ogawa, Prog. Surf. Sci. 56, 239 (1997).

[7] P. M. Echenique, R. Berndt, E. V. Chulkov, Th. Fauster, A. Goldmann and U. Höfer, Surf. Sci. Rep. 52, 219 (2004).

[8] K. Boger, M. Weinelt, and Th. Fauster, Phys. Rev. Lett. 92, 126803 (2004).

[9] J. Li, W. D. Schneider, R. Berndt, O. R. Bryant and S. Crampin, Phys. Rev. Lett. 81, 4464 (1998).

[10] J. Kliewer, R. Berndt, E. V. Chulkov, V. M. Silkin, P. M. Echenique and S. Crampin, Science 288, 1399 (2000).
[11] D. Wegner, A. Bauer, and G. Kaindl, Phys. Rev. Lett. 94, 126804 (2005).

[12] L. Limot, E. Pehlke, J. Kröger and R. Berndt, Phys. Rev. Lett. 94, 036805 (2005).

[13] L. Bürgi, O. Jeandupeux, H. Brune and K. Kern, Phys. Rev. Lett. 82, 4516 (1999).

[14] K. F. Braun and K. H. Rieder, Phys. Rev. Lett. 88, 096801 (2002).

[15] S. Crampin, J. Kröger, H. Jensen, and R. Berndt, cond-mat/0410542

[16] SAES Getters, Cologne, Germany.

[17] S. Å. Lindgren, L. Walldén, J. Rundgren, P. Westrin, and J. Neve, Phys. Rev. B 28, 6707 (1983).

[18] R. D. Diehl and R. McGrath, Surf. Sci. Rep. 23, 43 (1996).

[19] G. Kresse and J. Hafner, Phys. Rev. B 47, 588 (1993); ibid. 49, 14251 (1994); G. Kresse and J. Furthmüller, Comput. Mat. Sci. B 6, 15 (1996); Phys. Rev. B 54, 11169 (1996).

[20] P. E. Blöchl, Phys. Rev. B 50, 17953 (1994).

[21] J. P. Perdew, K. Burke, and Y. Wang, Phys. Rev. B 54, 16533 (1996).

[22] A. Partridge, G. J. Tatlock, F. M. Leibsle, C. F. J. Flipse, G. Hörmandiger, and J. B. Pendry, Phys. Rev. B 48, 8267 (1993).

[23] C. Corriol, A. Arnau, V. M. Silkin, E. V. Chulkov and P. M. Echenique (to be published).

[24] J. M. Carlsson and B. Hellsing, Phys. Rev. B 61, 13973 (2000).

[25] J. M. Soler, E. Artacho, J. D. Gale, A. García, J. Junquera, and D. Sánchez-Portal, J. Phys. C: Cond. Matter 14, 2745 (2002).

[26] We have used a double- $\zeta$ polarized basis sets of pseudoatomic orbitals. The radii of the orbitals for $\mathrm{Cu}$ were fixed with an energy shift [25] of $200 \mathrm{meV}$. The basis set of Cs and $\mathrm{Na}$ corresponds to a +1 ionic configuration, and the orbital radii were fixed respectively to 9 and 8 Bohrs. For Cs the $5 p$ electrons were included explicitly in the calculations.

[27] E. Artacho and F. Yndurain, Phys. Rev. B 44, 6169 (1991).

[28] We perform a fit to $\left[\left(\omega-\epsilon_{R}\right)^{2}+\left(\Gamma_{R} / 2\right)^{2}\right]^{-1}$ in the energy range of interest. In order to obtain convergence of the iterative solution of Eq. 2] it is necessary to evaluate the Green function outside the real axis. To this end, a small imaginary part is added to the energy $\omega+i \delta$. To estimate the physical (elastic) width $\Gamma_{\text {elastic }}$ of the QWS, this artificial width is substracted from the fitted value $\Gamma_{R}$. We have thus $\Gamma_{\text {elastic }}=\Gamma_{R}-2 \delta$. We have used three different values of $\delta,(1.0,0.5$ and $0.25 \mathrm{meV})$, obtaining identical values for $\Gamma_{\text {elastic }}$ within $\approx 0.1 \mathrm{meV}$.

[29] E. V. Chulkov, J. Kliewer, R. Berndt, V. M. Silkin, B. Hellsing, S. Crampin and P. M. Echenique, Phys. Rev. B 68, 195422 (2003).

[30] G. Witte and J. P. Toennies, Phys. Rev. B 62, 7771(R) (2000).

[31] G. Grimvall, in The Electron-Phonon Interaction in Metals, Selected Topics in Solid State Physics, edited by E. Wohlfarth (North-Holland, New York, 1981). 